\title{
A Thermodynamic Model on a Linear Rule between the Molal Concentration Exponential and the Osmotic Coefficients on Mole Fraction Base in Electrolyte Solutions and its Application
}

\author{
Zheng Fang
}

\author{
Chemistry and Chemical Engineering College, Central South University, Hunan 410083, China
}

\begin{abstract}
A linear rule is discovered. That is the osmotic coefficients on mole fraction base $(\omega)$ are linearly related to the molal concentration exponential $\left(m^{k_{2}}\right)$ in single electrolyte solutions. Based on this rule, a thermodynamic model is developed and successfully used to the single electrolytes with various valent types, such as uniunivalent, biunivalent, triunivalent, tetraunivalent, bibivalent, and tribivalent to predict their conventional osmotic coefficients on molal base $(\Phi)$, and also other properties, such as the relative molal vapor pressure lowering, the equivalent conductivity. Besides $m^{k_{2}}$, the $x^{k_{2}}$ (the concentration exponential of mole fraction of solute), $m^{k_{2}} \ln \left(m^{k_{2}}\right)$ and $x^{k_{2}} \ln \left(x^{k_{2}}\right)$ are also linearly related to $\omega$, respectively. They are all capable to be used to reproduce some properties of single electrolyte solutions. The examples with satisfied results have been given.
\end{abstract}

Keywords: Linear rule, single electrolyte solution, mixed electrolyte solution, osmotic coefficient, conductivity, solubility, Shannon information entropy.

\section{INTRODUCTION}

The electrolyte solutions widely exist in natural world, such as molten lava erupted from the volcano, sea water, and salt lake. In industry and in daily life, a large number of electrolytes are applied. Inside all organisms exists a variety of the electrolytes. Recently, we research the Peltier heats of the single electrode reaction [1, 2]. It also involves some thermodynamic properties of electrolytes and ions. So the close attention to the electrolyte solutions is still of high interest. Many equilibrium and transport properties, like osmotic coefficient, activity, surface tension, conductivity, ion transport number, etc., have been measured and identified by experiments. Some of rules, such as Faraday's laws of electrolysis, Kohlrausch's law of independent migration of ions, transport number law, etc., have been presented. A variety of theoretic models are continually proposed. It includes the Debye-Hückel interionic-attraction theory [3], ionic pairing [4], short-range force [5], ionic hydration [6], semi-empirical Pitzer equation [7] for single electrolytic system, some models for multicomponent system [8-11], MSA [12], MMSA [13] for certain particular systems, such as those of miscibility gap, liquid-liquid equilibria, even computer simulation [14] etc. Harned and Owen [15], Robinson and Stokes [16], and Bassett and Melchior [17] have excellently summarized and reviewed the developments. The

*Address correspondence to this author at the Chemistry and Chemical Engineering College, Central South University, Hunan 410083, China;

Tel: +86-0731-88660356; E-mail: zfang@csu.edu.cn progress in the theoretic respect of statistical physics has been also reviewed by García and Mosquera [18]. But the well known and the often used are the Pitzer or the extended Pitzer model on the molality scale and the Clegg-Pitzer-Brimblecombe model on the mole fraction scale [7, 19]. This paper will present a new discovery that the osmotic coefficients on mole fraction base are linearly related to the molal concentration exponential in aqueous electrolytes. And apply this rule to construct a thermodynamic model predicting some properties of electrolyte solutions.

\section{A LINEAR RULE}

The mole fraction of water in single electrolytic solution on a fully ionized basis, $x_{w}$ is known as

$x_{w}=n_{w} /\left(n_{w}+v m\right)$

The conventional osmotic coefficient, $\Phi$ is defined as [16]

$\Phi=-n_{w} \ln \left(a / a^{0}\right) /(v m)$

where $n_{w}$ is the number of moles of water, $v$, the number of ions into which a molecule of solute dissociates, $m$, the molal concentration of solute, $a$ and $a^{0}$ refer to the activity of water at $x_{w}<1$ and $x_{w}=1$, respectively. This is a dimensional quantity and it is on molal scale.

Now newly define the osmotic coefficient on mole fraction scale to be

$\omega=v \ln \left(a / a^{0}\right) / x^{k_{1}}$ 

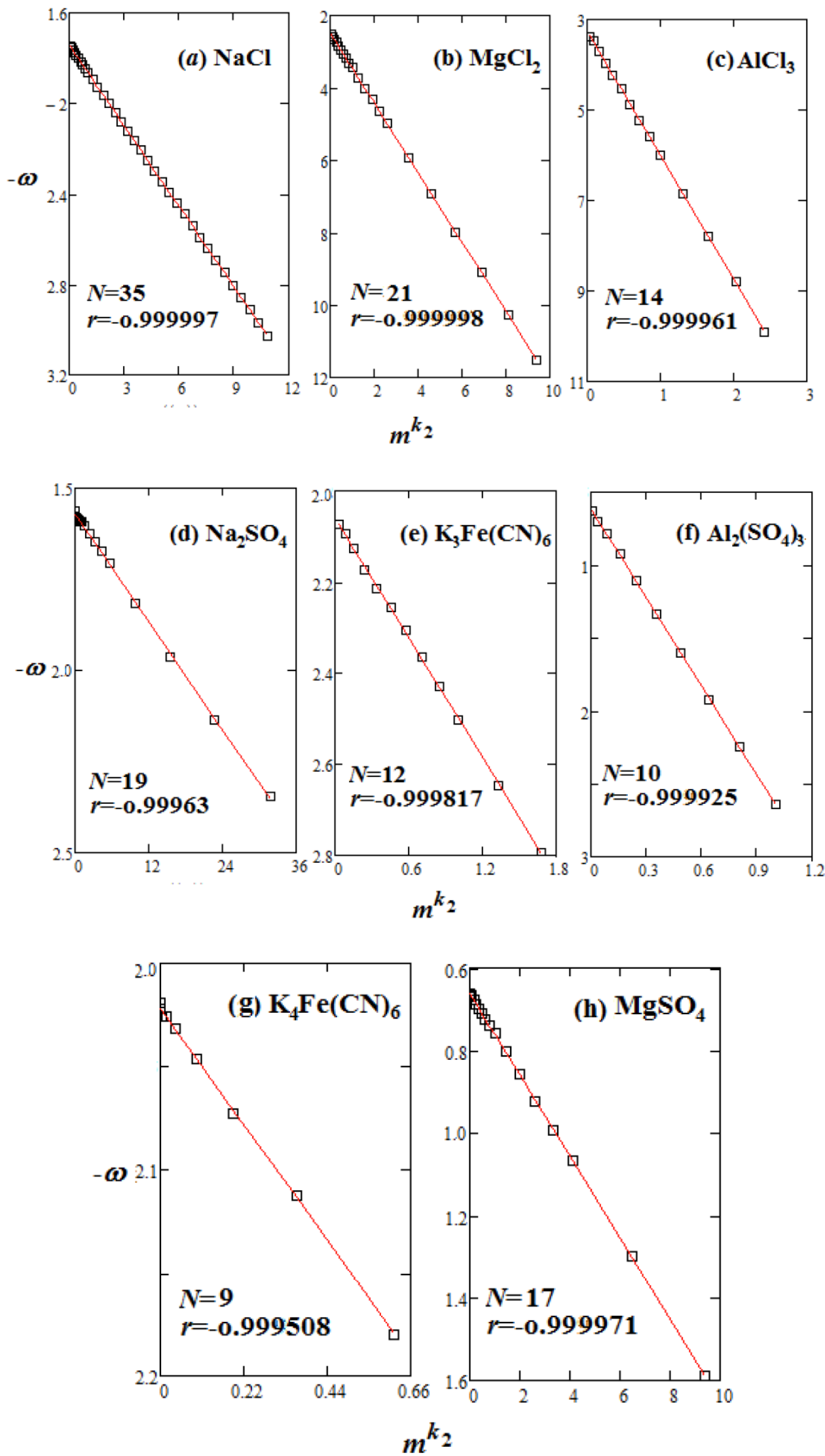

Figure 1: The linear relation between $\omega$ and $m^{k_{2}}$ for the electrolytes with various valent type; (a) uniunivalent, (b) biunivalent, (c) triunivalent, (d) unibivalent, (e) unitrivalent, (f) tribivalent, (g) unitetravalent and (h) bibivalent; $\square$, experimental point; presented straight-line of $\omega$ against $m^{k_{2}} ; N$, number of datum points and $r$, linear correlation coefficient. 
where $x$ is the mole fraction of solute, being $\left(1-x_{w}\right)$, and $k_{1}$ is a parameter to be determined. It has been proved that the function $\omega$ is similar to $\Phi$ [20], but the former is dimensionless, and the latter is just opposite, being of dimension.

The $\omega$ is very good linearly related to a probabilitydistribution function for solvent around the central ion hydrated, $\left(x_{w}\right)^{k_{2}} / \ln \left(x_{w}\right)$ [20].

However, the function $\omega$ is also linearly related to the molal concentration exponential, $m^{k_{2}}$, for various valent types of single electrolytes, such as uniunivalent, biunivalent (or unibivalent), triunivalent (or unitrivalent), tetraunivalent, bibivalent, and tribivalent ones. The certain examples are given in Figure 1, where it can be seen that the correlation coefficients for different valent types of single electrolytic solutions are marvelous.

\section{APPLICATION}

\subsection{The Osmotic Coefficients for Single Electrolytes}

Let

$h_{1}=m^{k_{2}}$

For single electrolytic system, as stated above,

$\omega=\alpha_{2}+\alpha_{1} h_{1}$

where $\alpha_{1}$ is slope and $\alpha_{2}$, intercept.

Combination of Eq. (2), Eq. (3), and Eq. (5) gives

$\Phi=-\left(\alpha_{2}+\alpha_{1} h_{1}\right) x^{k_{1}} n_{w} /\left(v^{2} m\right)$

This is an equation (called as the $h$ function model) for obtaining the osmotic coefficients for single electrolytes.

Now, we give some examples for different valent types of single electrolytes. The source data are taken from Ref. [16] under no special statement and others from Refs. [21-30]. The $k_{1}$ and $k_{2}$ are obtained and optimized by a gradual iteration with "Mathcad 14.0" software, and the $\alpha_{1}$ and the $\alpha_{2}$. are automatically identified subsequently. All parameters $\left(k_{2}, k_{1}, \alpha_{1}\right.$ and $\left.\alpha_{2}\right)$ and errors are listed in Tables 1-6, respectively. In order to evaluate the errors, one gives the variance, $\sigma$ as follows,

$\sigma=\sqrt{\frac{\sum_{i=1}^{N}\left(\Phi-\Phi_{c a l}\right)^{2}}{N}}$ where $N$ is the number of datum points, $\Phi$ and $\Phi_{\text {cal }}$ are, respectively, the osmotic coefficients determined experimentally and calculated by the models such as Pitzer or Eq. (6).

\subsection{Reproduction of another Properties}

Eq. (6) can be also related to some other properties which are dependent on the concentration, such as the relative molal vapor pressure lowering, $\delta$, equivalent conductivity, $\rho$ and solubility.

Take $\delta$ and $\rho$ of $\mathrm{KCl}$ for example. Replace $\Phi$ in Eq. (6) by $\delta$ or $\rho$. All the original data are taken from Ref. [16]. The parameters and errors are in Table 7. The average relative deviations (ARD) of them are all much less than $10^{-3}$, showing the excellent reproduction. The thermodynamic properties of electrolyte, like the boiling point elevation, lowering of freezing point, etc., are related to the change in the vapor pressure, so these could be reproduced well based on this linear rule.

The present model can also be used to reproduce the solubility accurately. Take the solubility of $\mathrm{MgCl}_{2}$ in the $\mathrm{HCl}-\mathrm{LiCl}-\mathrm{MgCl}_{2}-\mathrm{H}_{2} \mathrm{O}$ mixture at $273 \mathrm{~K}$ for an example [31]. The chemical potential of $\mathrm{MgCl}_{2}$ in the quaternary system changes no longer owing to its saturation, not disturbing the ion distribution. The concentration of the mixture is simply considered as $m_{\text {mix }}=\left(2 m_{\mathrm{HCl}}+2 m_{\mathrm{LiCl}}\right)$ and $x_{\mathrm{H}_{2} \mathrm{O}}=n_{w} /\left(n_{w}+m_{\text {mix }}\right)$. The results by the present model and a BET equation improved by Ref. [32] are listed in Table 8, where it can be seen that the present results are better than the improved BET.

\section{DISCUSSIONS}

In this paper, the functions $\omega$ and $m^{k_{2}}$ are introduced. Similar to the conventional $\Phi$ that is considered as a potential energy, the $\omega$ can also be thought as a dimensionless-thermodynamic potential [20]. The $h_{1}=m^{k_{2}}$ can be seen a comprehensive effects of a variety of forces. These effects, such as the longrange, the short-range and the higher-order forces, in general, are simply summarized together to describe the total interionic interactions. Actually, they do not include all effects, e.g. the interactions between different forces. Suppose that all forces and their interactions are taken into account to be related to the ionic strength exponential, $I^{k_{2}}$. It could represent a whole outcome of the density and the intensity of all the ions. Therefore, we can say that the $I^{k_{2}}$ integrates a 
Table 1: Parameters and the Errors for Prediction of the Osmotic Coefficients of Uniunivalent Electrolytes by the $h$ Function Model

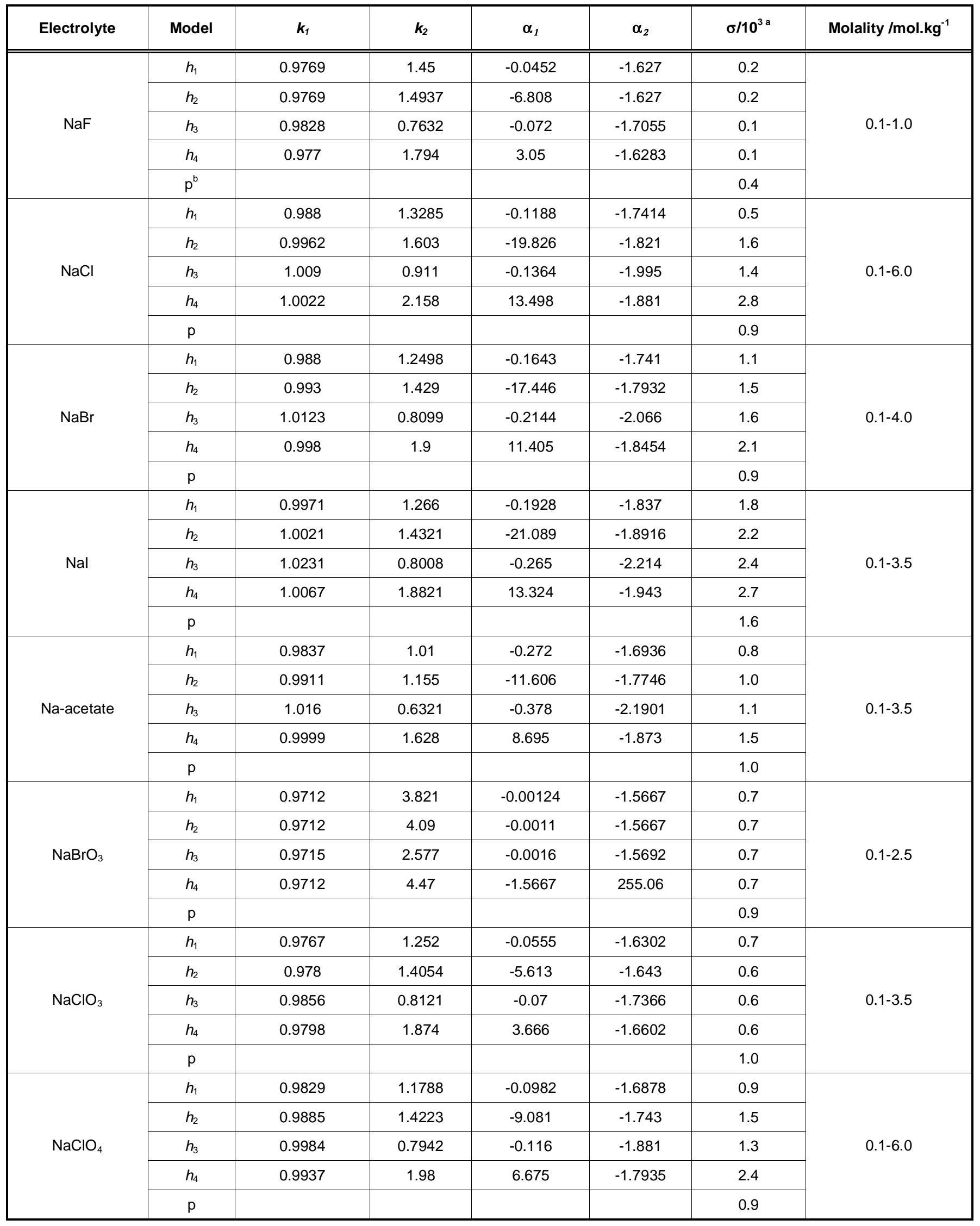


(Table 1). Continued.

\begin{tabular}{|c|c|c|c|c|c|c|c|}
\hline Electrolyte & Model & $k_{1}$ & $k_{2}$ & $\alpha_{1}$ & $\alpha_{2}$ & $\sigma / 10^{3 a}$ & Molality /mol.kg ${ }^{-1}$ \\
\hline \multirow{5}{*}{ NaCNS } & $h_{1}$ & 0.991 & 1.11 & -0.1735 & -1.7718 & 0.9 & \multirow{5}{*}{$0.1-4.0$} \\
\hline & $h_{2}$ & 0.996 & 1.271 & -10.842 & -1.826 & 1.3 & \\
\hline & $h_{3}$ & 1.0135 & 0.7134 & -0.2274 & -2.0971 & 1.3 & \\
\hline & $h_{4}$ & 1.0018 & 1.7621 & 7.851 & -1.8904 & 1.9 & \\
\hline & $p$ & & & & & 1.0 & \\
\hline \multirow{5}{*}{ Na formate } & $h_{1}$ & 0.982 & 0.98 & -0.1584 & -1.6709 & 0.6 & \multirow{5}{*}{$0.1-3.5$} \\
\hline & $h_{2}$ & 0.9861 & 1.121 & -5.999 & -1.7154 & 0.8 & \\
\hline & $h_{3}$ & 1.001 & 0.6197 & -0.2105 & -1.9495 & 0.8 & \\
\hline & $h_{4}$ & 0.9912 & 1.594 & 4.5437 & -1.7706 & 1.1 & \\
\hline & $p$ & & & & & 1.2 & \\
\hline \multirow{5}{*}{$\mathrm{NaOH}$} & $h_{1}$ & 1.0121 & 1.5987 & -0.0907 & -1.9752 & 2.3 & \multirow{5}{*}{$0.1-6.0$} \\
\hline & $h_{2}$ & 1.0176 & 1.908 & -42.175 & -2.035 & 2.9 & \\
\hline & $h_{3}$ & 1.0285 & 1.1258 & -0.0978 & -2.1814 & 3.2 & \\
\hline & $h_{4}$ & 1.022 & 2.453 & 25.118 & -2.0812 & 3.8 & \\
\hline & $p$ & & & & & 8.8 & \\
\hline \multirow{5}{*}{$\mathrm{KF}$} & $h_{1}$ & 0.9798 & 1.2344 & -0.1454 & -1.656 & 1.2 & \multirow{5}{*}{$0.1-3.5$} \\
\hline & $h_{2}$ & 0.9844 & 1.4107 & -14.5290 & -1.7015 & 1.7 & \\
\hline & $h_{3}$ & 1.002 & 0.7966 & -0.1905 & -1.9396 & 1.7 & \\
\hline & $h_{4}$ & 0.9856 & 1.8 & 8.415 & -1.7154 & 1.4 & \\
\hline & $p$ & & & & & 1.0 & \\
\hline \multirow{5}{*}{$\mathrm{KCl}$} & $h_{1}$ & 0.9771 & 1.1878 & -0.0918 & -1.6293 & 0.3 & \multirow{5}{*}{$0.1-4.5$} \\
\hline & $h_{2}$ & 0.9809 & 1.3848 & -8.0535 & -1.666 & 0.5 & \\
\hline & $h_{3}$ & 0.9915 & 0.7805 & -0.1139 & -1.8057 & 0.5 & \\
\hline & $h_{4}$ & 0.9842 & 1.878 & 5.5347 & -1.6978 & 0.9 & \\
\hline & $p$ & & & & & 0.9 & \\
\hline \multirow{5}{*}{$\mathrm{KBr}$} & $h_{1}$ & 0.9759 & 1.0948 & -0.115 & -1.6175 & 0.5 & \multirow{5}{*}{$0.1-5.5$} \\
\hline & $h_{2}$ & 0.9816 & 1.31 & -7.496 & -1.6733 & 0.7 & \\
\hline & $h_{3}$ & 0.9932 & 0.727 & -0.1398 & -1.8354 & 0.6 & \\
\hline & $h_{4}$ & 0.9872 & 1.8525 & 5.687 & -1.7277 & 1.4 & \\
\hline & $p$ & & & & & 0.6 & \\
\hline \multirow{5}{*}{$\mathrm{KI}$} & $h_{1}$ & 0.972 & 0.948 & -0.167 & -1.5803 & 0.9 & \multirow{5}{*}{$0.1-4.5$} \\
\hline & $h_{2}$ & 0.9787 & 1.122 & -5.976 & -1.6484 & 0.6 & \\
\hline & $h_{3}$ & 0.9935 & 0.6126 & -0.2138 & -1.8774 & 0.8 & \\
\hline & $h_{4}$ & 0.9855 & 1.633 & 4.762 & -1.718 & 0.6 & \\
\hline & $p$ & & & & & 0.7 & \\
\hline \multirow{5}{*}{$\mathrm{KNO}_{3}$} & $h_{1}$ & 0.9772 & 0.594 & 0.1989 & -1.649 & 1.1 & \multirow{5}{*}{$0.1-3.5$} \\
\hline & $h_{2}$ & 0.977 & 0.6534 & 1.702 & -1.6385 & 1.0 & \\
\hline & $h_{3}$ & 0.96 & 0.396 & 0.2249 & -1.3686 & 1.1 & \\
\hline & $h_{4}$ & 0.966 & 1.207 & -1.6426 & -1.5124 & 0.9 & \\
\hline & $p$ & & & & & 2.7 & \\
\hline
\end{tabular}


(Table 1). Continued.

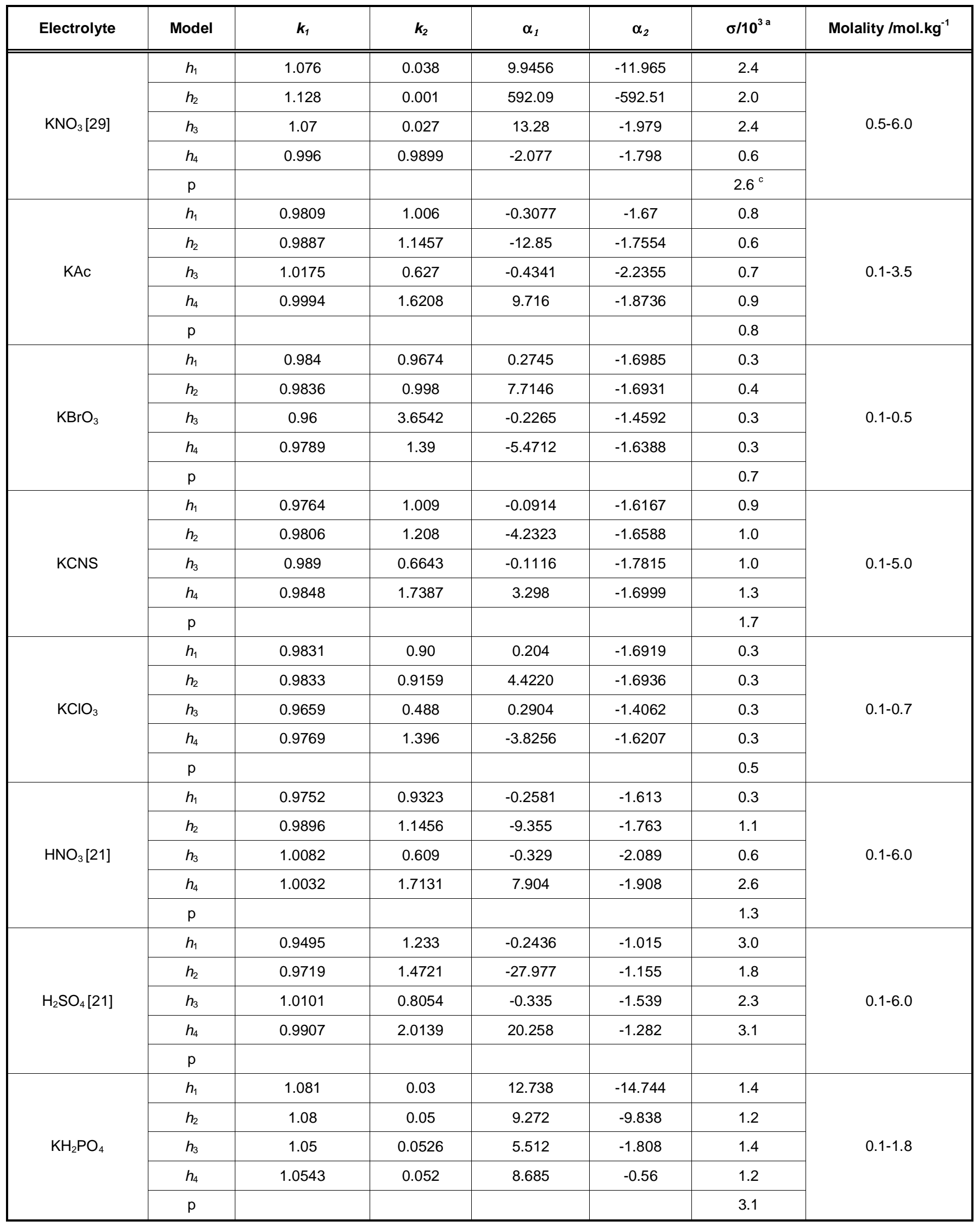


(Table 1). Continued.

\begin{tabular}{|c|c|c|c|c|c|c|c|}
\hline Electrolyte & Model & $k_{1}$ & $k_{2}$ & $\alpha_{1}$ & $\alpha_{2}$ & $\sigma / 10^{3 a}$ & Molality /mol.kg ${ }^{-1}$ \\
\hline \multirow{5}{*}{$\mathrm{KOH}$} & $h_{1}$ & 0.9926 & 1.2107 & -0.253 & -1.772 & 2.3 & \multirow{5}{*}{$0.1-6.0$} \\
\hline & $h_{2}$ & 1.007 & 1.4552 & -26.638 & -1.923 & 3.8 & \\
\hline & $h_{3}$ & 1.03 & 0.8043 & -0.321 & -2.293 & 3.5 & \\
\hline & $h_{4}$ & 1.0188 & 2.004 & 19.345 & -2.053 & 5.8 & \\
\hline & $p$ & & & & & 9.5 & \\
\hline \multirow{5}{*}{$\mathrm{LiCl}$} & $h_{1}$ & 1.0051 & 1.3476 & -0.2212 & -1.919 & 2.3 & \multirow{5}{*}{$0.1-6.0$} \\
\hline & $h_{2}$ & 1.0174 & 1.6095 & -39.102 & -2.0544 & 4.1 & \\
\hline & $h_{3}$ & 1.0395 & 0.9112 & -0.2699 & -2.4 & 4.0 & \\
\hline & $h_{4}$ & 1.0283 & 2.1585 & 26.551 & -2.176 & 6.1 & \\
\hline & $p$ & & & & & 1.9 & \\
\hline \multirow{5}{*}{$\mathrm{LiBr}$} & $h_{1}$ & 0.9817 & 1.1777 & -0.3323 & -1.687 & 1.0 & \multirow{5}{*}{$0.1-2.5$} \\
\hline & $h_{2}$ & 0.987 & 1.295 & -24.142 & -1.7436 & 0.7 & \\
\hline & $h_{3}$ & 1.0242 & 0.7151 & -0.5017 & -2.328 & 0.6 & \\
\hline & $h_{4}$ & 1.208 & 0.3056 & -29.775 & -15.265 & 1.2 & \\
\hline & $p$ & & & & & 2.0 & \\
\hline \multirow{5}{*}{ Lil } & $h_{1}$ & 1.0238 & 1.48 & -0.2477 & -2.171 & 1.8 & \multirow{5}{*}{$0.1-3.0$} \\
\hline & $h_{2}$ & 1.028 & 1.6418 & -56.6 & -2.224 & 2.2 & \\
\hline & $h_{3}$ & 1.0555 & 0.9315 & -0.3516 & -2.688 & 2.8 & \\
\hline & $h_{4}$ & 1.0324 & 2.072 & 31.377 & -2.28 & 2.7 & \\
\hline & $p$ & & & & & 6.0 & \\
\hline \multirow{5}{*}{ LiAc } & $h_{1}$ & 0.974 & 0.9867 & -0.23 & -1.5977 & 0.5 & \multirow{5}{*}{$0.1-4.0$} \\
\hline & $h_{2}$ & 0.9815 & 1.1427 & -9.213 & -1.6752 & 0.4 & \\
\hline & $h_{3}$ & 1.0034 & 0.6277 & -0.3081 & -2.0166 & 0.5 & \\
\hline & $h_{4}$ & 0.9905 & 1.6377 & 7.1429 & -1.7693 & 0.9 & \\
\hline & $p$ & & & & & & \\
\hline \multirow{5}{*}{$\mathrm{LiClO}_{4}$} & $h_{1}$ & 0.9995 & 1.196 & -0.3377 & -1.881 & 1.6 & \multirow{5}{*}{$0.1-4.0$} \\
\hline & $h_{2}$ & 1.0091 & 1.367 & -29.43 & -1.9913 & 1.6 & \\
\hline & $h_{3}$ & 1.0414 & 0.755 & -0.4794 & -2.551 & 1.9 & \\
\hline & $h_{4}$ & 1.0194 & 1.8443 & 20.148 & -2.113 & 2.2 & \\
\hline & $p$ & & & & & 2.2 & \\
\hline \multirow{5}{*}{$\mathrm{LiNO}_{3}$} & $h_{1}$ & 0.9746 & 0.9863 & -0.2927 & -1.603 & 1.8 & \multirow{5}{*}{$0.1-6.0$} \\
\hline & $h_{2}$ & 0.9903 & 1.1973 & -12.952 & -1.7644 & 1.2 & \\
\hline & $h_{3}$ & 1.0137 & 0.6413 & -0.3819 & -2.16 & 1.5 & \\
\hline & $h_{4}$ & 1.0058 & 1.757 & 10.6617 & -1.929 & 2.4 & \\
\hline & $p$ & & & & & 1.1 & \\
\hline \multirow{5}{*}{$\mathrm{LiOH}(\mathrm{L})$} & $h_{1}$ & 0.9905 & 4.4322 & -0.0159 & -1.704 & 1.0 & \multirow{5}{*}{$0.1-1.0$} \\
\hline & $h_{2}$ & 0.9907 & 5.5431 & $-1.9 \times 10^{6}$ & -1.7059 & 1.0 & \\
\hline & $h_{3}$ & 0.995 & 2.133 & -0.0348 & -1.7474 & 0.8 & \\
\hline & $h_{4}$ & 0.9906 & 5.4321 & $7.3 \times 10^{4}$ & -1.705 & 1.0 & \\
\hline & $p$ & & & & & & \\
\hline
\end{tabular}


(Table 1). Continued.

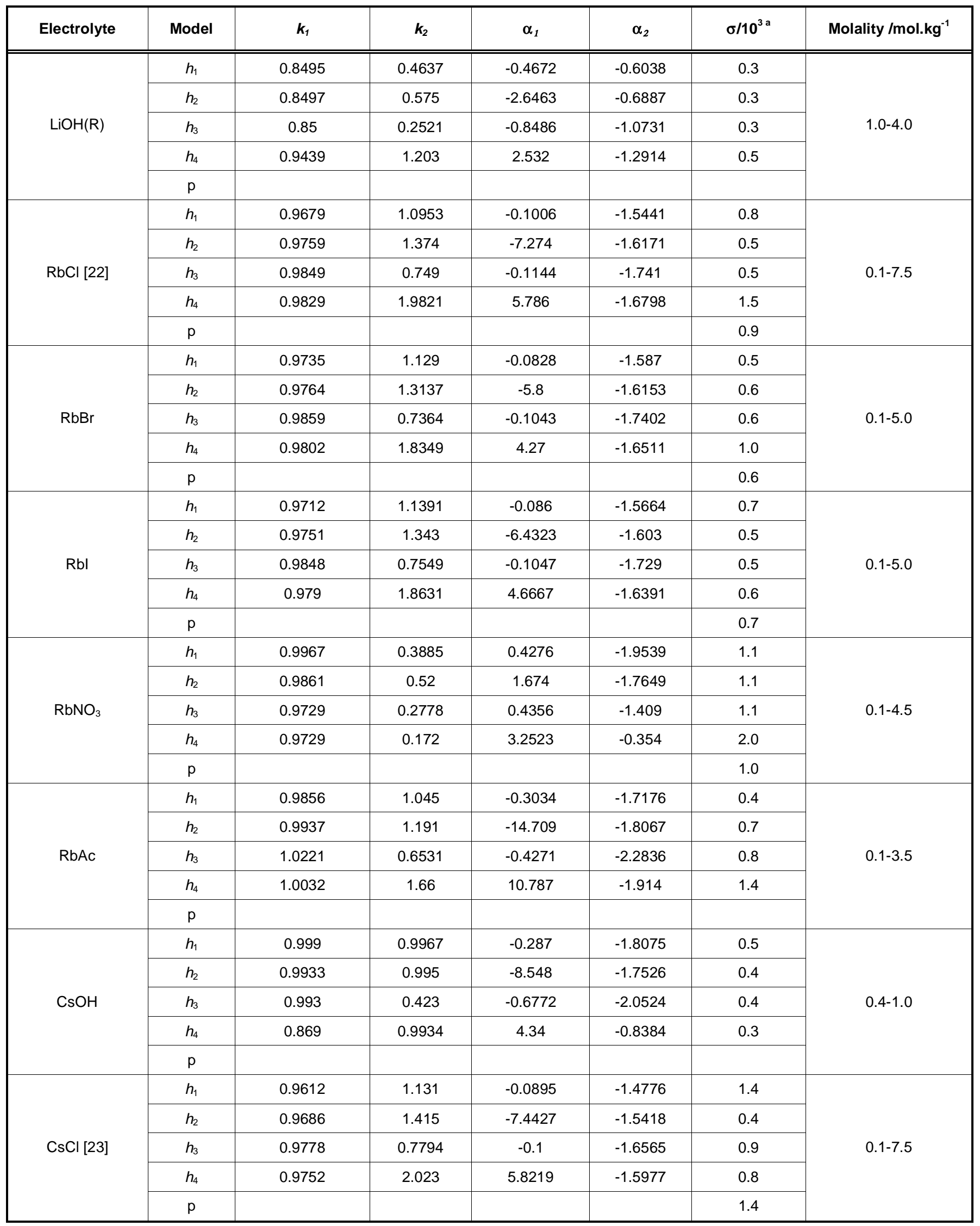


(Table 1). Continued.

\begin{tabular}{|c|c|c|c|c|c|c|c|}
\hline Electrolyte & Model & $k_{1}$ & $k_{2}$ & $\alpha_{1}$ & $\alpha_{2}$ & $\sigma / 10^{3 a}$ & Molality /mol. kg ${ }^{-1}$ \\
\hline \multirow{5}{*}{ CsAc } & $h_{1}$ & 0.9869 & 1.045 & -0.3035 & -1.7339 & 1.0 & \multirow{5}{*}{$0.1-3.5$} \\
\hline & $h_{2}$ & 0.9945 & 1.1864 & -14.585 & -1.818 & 1.2 & \\
\hline & $h_{3}$ & 1.0229 & 0.6499 & -0.4304 & -2.2985 & 1.2 & \\
\hline & $h_{4}$ & 1.0044 & 1.6574 & 10.7416 & -1.93 & 1.6 & \\
\hline & $p$ & & & & & & \\
\hline \multirow{5}{*}{$\mathrm{CsNO}_{3}$} & $h_{1}$ & 0.9757 & 0.5511 & 0.2406 & -1.6466 & 0.6 & \multirow{5}{*}{$0.1-1.4$} \\
\hline & $h_{2}$ & 0.9774 & 0.5585 & 1.636 & -1.6644 & 0.6 & \\
\hline & $h_{3}$ & 0.9553 & 0.3778 & 0.2601 & -1.3128 & 0.6 & \\
\hline & $h_{4}$ & 0.9653 & 1.059 & -1.531 & -1.5127 & 0.7 & \\
\hline & $p$ & & & & & 1.4 & \\
\hline \multirow{5}{*}{$\mathrm{NH}_{4} \mathrm{Cl}$} & $h_{1}$ & 0.9646 & 0.909 & -0.1398 & -1.5105 & 1.4 & \multirow{5}{*}{$0.1-6.0$} \\
\hline & $h_{2}$ & 0.9731 & 1.1238 & -4.61 & -1.5918 & 0.9 & \\
\hline & $h_{3}$ & 0.9837 & 0.5976 & -0.1718 & -1.759 & 1.1 & \\
\hline & $h_{4}$ & 0.98 & 1.6796 & 3.87 & -1.6587 & 0.6 & \\
\hline & $p$ & & & & & 0.9 & \\
\hline \multirow{5}{*}{$\mathrm{NH}_{4} \mathrm{NO}_{3}$} & $h_{1}$ & 0.824 & 0.1073 & -1.2067 & 0.2639 & 0.9 & \multirow{5}{*}{$0.1-6.0$} \\
\hline & $h_{2}$ & 0.824 & 0.1489 & -1.5132 & -0.0255 & 1.4 & \\
\hline & $h_{3}$ & 0.7339 & 0.0946 & -1.645 & -0.6966 & 0.8 & \\
\hline & $h_{4}$ & 0.9861 & 0.583 & -0.4216 & -1.7419 & 0.8 & \\
\hline & $p$ & & & & & 1.4 & \\
\hline \multirow{5}{*}{$\mathrm{HCl}$} & $h_{1}$ & 0.9914 & 1.1992 & -0.3078 & -1.782 & 0.9 & \multirow{5}{*}{$0.1-6.0$} \\
\hline & $h_{2}$ & 1.0082 & 1.4363 & -30.846 & -1.961 & 2.7 & \\
\hline & $h_{3}$ & 1.0362 & 0.7914 & -0.4002 & -2.426 & 2.3 & \\
\hline & $h_{4}$ & 1.0235 & 1.9857 & 22.681 & -2.132 & 5.3 & \\
\hline & $p$ & & & & & 4.9 & \\
\hline \multirow{5}{*}{$\mathrm{HBr}$} & $h_{1}$ & 0.9961 & 1.25 & -0.3534 & -1.8396 & 1.4 & \multirow{5}{*}{$0.1-3.0$} \\
\hline & $h_{2}$ & 1.0028 & 1.39 & -34.802 & -1.9155 & 1.8 & \\
\hline & $h_{3}$ & 1.0407 & 0.7697 & -0.5281 & -2.5455 & 2.1 & \\
\hline & $h_{4}$ & 1.0114 & 1.831 & 22.2157 & -2.0146 & 2.4 & \\
\hline & $p$ & & & & & 1.6 & \\
\hline \multirow{5}{*}{$\mathrm{HI}$} & $h_{1}$ & 1.0075 & 1.2084 & -0.3948 & -1.969 & 1.1 & \multirow{5}{*}{$0.1-3.0$} \\
\hline & $h_{2}$ & 1.0143 & 1.3463 & -33.507 & -2.0533 & 1.6 & \\
\hline & $h_{3}$ & 1.0532 & 0.7465 & -0.585 & -2.754 & 1.9 & \\
\hline & $h_{4}$ & 1.0235 & 1.787 & 21.838 & -2.1676 & 2.3 & \\
\hline & $p$ & & & & & 2.2 & \\
\hline \multirow{5}{*}{$\mathrm{HClO}_{4}$} & $h_{1}$ & 1.0019 & 1.39 & -0.2665 & -1.905 & 2.4 & \multirow{5}{*}{$0.1-5.5$} \\
\hline & $h_{2}$ & 1.015 & 1.635 & -53.284 & -2.048 & 4.7 & \\
\hline & $h_{3}$ & 1.0432 & 0.9275 & -0.341 & -2.49 & 4.5 & \\
\hline & $h_{4}$ & 1.0265 & 2.161 & 34.82 & -2.178 & 6.9 & \\
\hline & $p$ & & & & & 2.5 & \\
\hline
\end{tabular}


(Table 1). Continued.

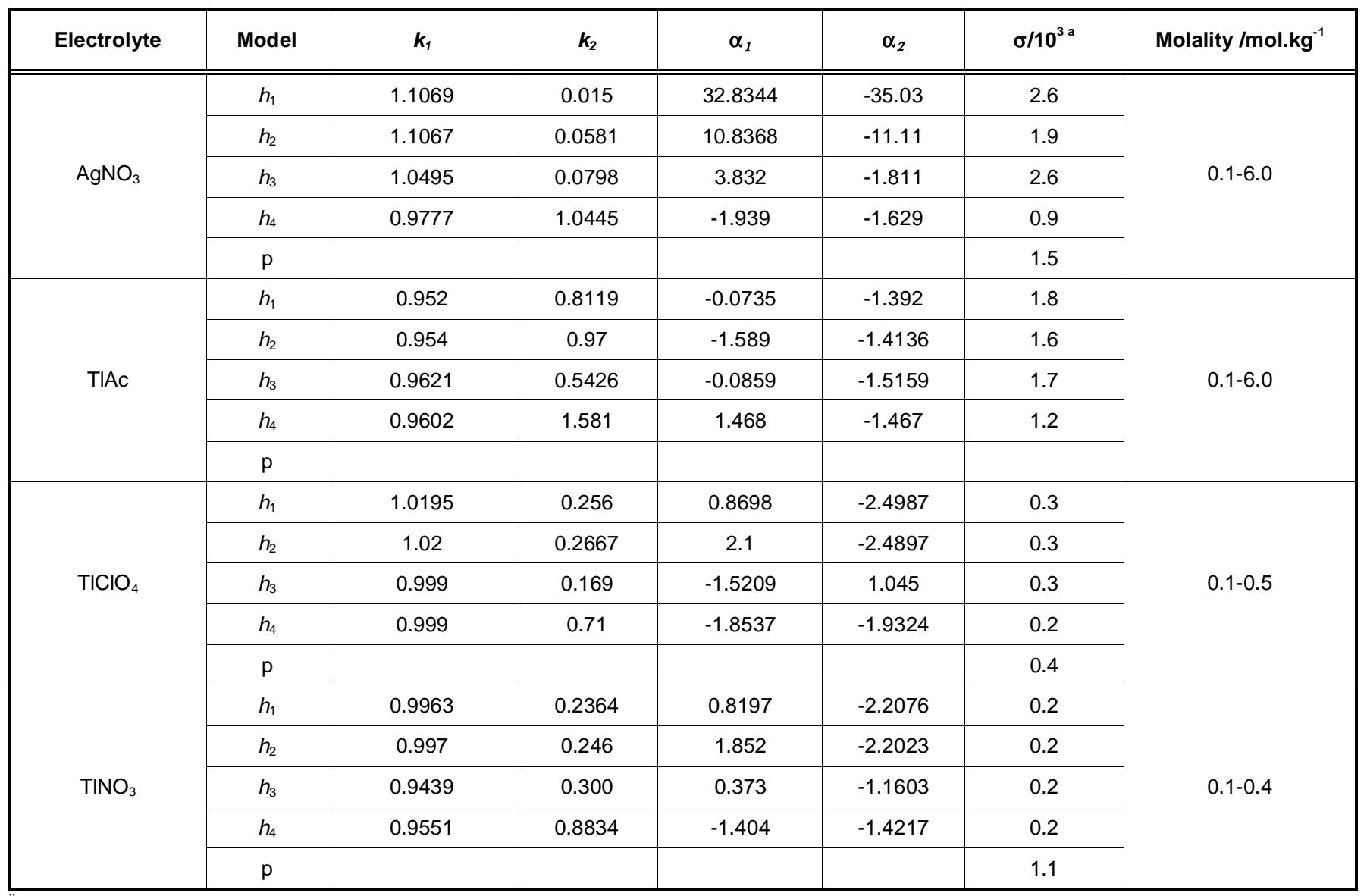

${ }^{a} \sigma$ is variance calculated on Eq. (7).

p represents Pitzer model (the same below).

'This datum is according to Extended Pitzer equation.

Table 2: Parameters and the Errors for Prediction of the Osmotic Coefficients of Biunivalent Electrolytes by the $h$ Function Model

\begin{tabular}{|c|c|c|c|c|c|c|c|}
\hline Electrolyte & Model & $k_{1}$ & $k_{2}$ & $\alpha_{1}$ & $\alpha_{2}$ & $\sigma / 10^{3}$ & $\begin{array}{l}\text { Molality } \\
/ \mathrm{mol}^{-} \mathrm{kg}^{-1}\end{array}$ \\
\hline \multirow{3}{*}{$\mathrm{BaAc}_{2}$} & $h_{1}$ & 0.6587 & 0.4941 & -0.8786 & -0.1234 & 1.4 & \multirow{3}{*}{$0.1-1.2$} \\
\hline & $h_{3}$ & 0.6982 & 0.2048 & -2.1348 & -1.1265 & 1.0 & \\
\hline & $h_{4}$ & 0.8692 & 0.8241 & 4.265 & -0.9706 & 0.8 & \\
\hline \multirow{4}{*}{$\mathrm{BaCl}_{2}$} & $h_{1}$ & 0.9635 & 1.1267 & -0.5969 & -2.056 & 1.1 & \multirow{4}{*}{$0.1-1.8$} \\
\hline & $h_{2}$ & 0.9701 & 1.253 & -23.414 & -2.1402 & 1.0 & \\
\hline & $h_{3}$ & 1.017 & 0.643 & -0.989 & -3.1108 & 1.1 & \\
\hline & $h_{4}$ & 0.9788 & 1.699 & 16.064 & -2.256 & 1.1 & \\
\hline \multirow[t]{3}{*}{$\mathrm{BaBr}_{2}$} & $h_{3}$ & 1.0429 & 0.6797 & -1.319 & -3.642 & 1.2 & \multirow[t]{3}{*}{$0.1-2.0$} \\
\hline & $h_{4}$ & 0.9964 & 1.7791 & 25.28 & -2.495 & 1.3 & \\
\hline & $p$ & & & & & 1.4 & \\
\hline
\end{tabular}


(Table 2). Continued.

\begin{tabular}{|c|c|c|c|c|c|c|c|}
\hline Electrolyte & Model & $k_{1}$ & $k_{2}$ & $\alpha_{1}$ & $\alpha_{2}$ & $\sigma / 10^{3}$ & $\begin{array}{l}\text { Molality } \\
/ \mathrm{mol}^{-1} \mathbf{k g}^{-1}\end{array}$ \\
\hline \multirow{5}{*}{$\mathrm{Bal}_{2}$} & $h_{1}$ & 0.9909 & 1.1899 & -1.1411 & -2.4256 & 1.8 & \multirow{5}{*}{$0.1-2.0$} \\
\hline & $h_{2}$ & 1.011 & 1.3621 & -60.803 & -2.721 & 2.0 & \\
\hline & $h_{3}$ & 1.0814 & 0.6827 & -2.03 & -4.665 & 2.2 & \\
\hline & $h_{4}$ & 1.0227 & 1.7922 & 38.78 & -2.912 & 2.5 & \\
\hline & $p$ & & & & & 2.5 & \\
\hline \multirow{5}{*}{$\mathrm{Ba}\left(\mathrm{NO}_{3}\right)_{2}$} & $h_{1}$ & 1.08 & 0.101 & 6.5925 & -8.758 & 0.5 & \multirow{5}{*}{$0.1-0.4$} \\
\hline & $h_{2}$ & 1.08 & 0.12 & 8.226 & -7.926 & 0.5 & \\
\hline & $h_{3}$ & 1.04 & 0.1011 & 5.114 & -1.9233 & 0.5 & \\
\hline & $h_{4}$ & 0.97 & 0.9698 & -3.342 & -2.0947 & 0.6 & \\
\hline & $p$ & & & & & 1.3 & \\
\hline \multirow{5}{*}{$\mathrm{CaCl}_{2}$} & $h_{1}$ & 0.992 & 1.38 & -0.7923 & -2.4358 & 1.2 & \multirow{5}{*}{$0.1-2.5$} \\
\hline & $h_{2}$ & 1.0059 & 1.5787 & -79.77 & -2.629 & 2.0 & \\
\hline & $h_{3}$ & 1.0686 & 0.82 & -1.328 & -4.049 & 2.4 & \\
\hline & $h_{4}$ & 1.017 & 2.0456 & 48.79 & -2.79 & 2.9 & \\
\hline & $p$ & & & & & 3.7 & \\
\hline \multirow{5}{*}{$\mathrm{CaBr}_{2}$} & $h_{1}$ & 0.9891 & 1.3 & -1.055 & -2.405 & 1.2 & \multirow{5}{*}{$0.1-2.0$} \\
\hline & $h_{2}$ & 0.9993 & 1.4438 & -74.0 & -2.552 & 1.8 & \\
\hline & $h_{3}$ & 1.0769 & 0.732 & -1.946 & -4.492 & 2.1 & \\
\hline & $h_{4}$ & 1.016 & 1.903 & 47.807 & -2.799 & 2.4 & \\
\hline & $p$ & & & & & 1.4 & \\
\hline \multirow{5}{*}{$\mathrm{Cal}_{2}$} & $h_{1}$ & 0.997 & 1.2914 & -1.263 & -2.55 & 1.1 & \multirow{5}{*}{$0.1-2.0$} \\
\hline & $h_{2}$ & 1.0104 & 1.4395 & -87.421 & -2.754 & 1.3 & \\
\hline & $h_{3}$ & 1.0936 & 0.721 & -2.399 & -5.081 & 1.5 & \\
\hline & $h_{4}$ & 1.0252 & 1.8797 & 54.936 & -2.993 & 1.9 & \\
\hline & $p$ & & & & & 1.5 & \\
\hline \multirow{5}{*}{$\mathrm{Ca}\left(\mathrm{ClO}_{4}\right)_{2}$} & $h_{1}$ & 1.011 & 1.3323 & -1.2174 & -2.763 & 0.8 & \multirow{5}{*}{$0.1-2.0$} \\
\hline & $h_{2}$ & 1.0225 & 1.4838 & -95.939 & -2.9507 & 1.3 & \\
\hline & $h_{3}$ & 1.1 & 0.7457 & -2.279 & -5.1862 & 1.8 & \\
\hline & $h_{4}$ & 1.0353 & 1.923 & 58.744 & -3.169 & 2.0 & \\
\hline & $p$ & & & & & 5.6 & \\
\hline \multirow{5}{*}{$\mathrm{CuCl}_{2}$} & $h_{1}$ & 0.84 & 0.6579 & -0.977 & -0.891 & 1.6 & \multirow{5}{*}{$0.1-2.0$} \\
\hline & $h_{2}$ & 0.8827 & 0.7549 & -8.514 & -1.218 & 1.4 & \\
\hline & $h_{3}$ & 0.9455 & 0.3545 & -1.764 & -2.556 & 1.4 & \\
\hline & $h_{4}$ & 0.9323 & 1.237 & 8.056 & -1.709 & 1.2 & \\
\hline & $\mathrm{p}$ & & & & & 4.3 & \\
\hline \multirow{5}{*}{$\mathrm{MnCl}_{2}$} & $h_{1}$ & 0.9571 & 1.100 & -0.849 & -1.996 & 2.3 & \multirow{5}{*}{$0.1-2.0$} \\
\hline & $h_{2}$ & 0.969 & 1.2353 & -31.497 & -2.146 & 2.0 & \\
\hline & $h_{3}$ & 1.0338 & 0.6313 & -1.457 & -3.573 & 2.0 & \\
\hline & $h_{4}$ & 0.9822 & 1.6777 & 21.86 & -2.321 & 1.6 & \\
\hline & $p$ & & & & & 3.6 & \\
\hline
\end{tabular}


(Table 2). Continued.

\begin{tabular}{|c|c|c|c|c|c|c|c|}
\hline Electrolyte & Model & $k_{1}$ & $k_{2}$ & $\alpha_{1}$ & $\alpha_{2}$ & $\sigma / 10^{3}$ & $\begin{array}{l}\text { Molality } \\
/ \mathrm{mol}^{-1} \mathrm{~kg}^{-1}\end{array}$ \\
\hline \multirow{5}{*}{$\mathrm{SrCl}_{2}[24]$} & $h_{1}$ & 0.9824 & 1.3543 & -0.713 & -2.302 & 3.3 & \multirow{5}{*}{$0.1-3.8$} \\
\hline & $h_{2}$ & 1.0052 & 1.6157 & -75.253 & -2.6 & 2.5 & \\
\hline & $h_{3}$ & 1.0596 & 0.8434 & -1.1026 & -3.7826 & 3.1 & \\
\hline & $h_{4}$ & 1.0225 & 2.1554 & 50.724 & -2.844 & 3.3 & \\
\hline & $p$ & & & & & 3.4 & \\
\hline \multirow{5}{*}{$\mathrm{FeCl}_{2}$} & $h_{1}$ & 0.9722 & 1.2344 & -0.892 & -2.179 & 1.6 & \multirow{5}{*}{$0.1-2.0$} \\
\hline & $h_{2}$ & 0.9834 & 1.381 & -51.365 & -2.326 & 1.2 & \\
\hline & $h_{3}$ & 1.0513 & 0.6973 & -1.599 & -3.885 & 1.1 & \\
\hline & $h_{4}$ & 0.9956 & 1.822 & 33.06 & -2.4943 & 0.9 & \\
\hline & $p$ & & & & & 1.7 & \\
\hline \multirow{5}{*}{$\mathrm{MgBr}_{2}$} & $h_{1}$ & 0.9894 & 1.26 & -1.31 & -2.422 & 1.0 & \multirow{5}{*}{$0.1-2.0$} \\
\hline & $h_{2}$ & 1.0034 & 1.402 & -81.38 & -2.628 & 1.2 & \\
\hline & $h_{3}$ & 1.089 & 0.695 & -2.544 & -5.018 & 1.6 & \\
\hline & $h_{4}$ & 1.02 & 1.845 & 52.48 & -2.889 & 1.9 & \\
\hline & $p$ & & & & & 3.9 & \\
\hline \multirow{5}{*}{$\mathrm{MgCl}_{2}$} & $h_{1}$ & 0.998 & 1.402 & -0.949 & -2.533 & 1.0 & \multirow{5}{*}{$0.1-2.0$} \\
\hline & $h_{2}$ & 1.007 & 1.558 & -93.202 & -2.666 & 0.9 & \\
\hline & $h_{3}$ & 1.0794 & 0.7946 & -1.728 & -4.435 & 1.3 & \\
\hline & $h_{4}$ & 1.0169 & 1.9929 & 54.521 & -2.816 & 1.3 & \\
\hline & $p$ & & & & & 1.7 & \\
\hline \multirow{5}{*}{$\mathrm{Mgl}_{2}$} & $h_{1}$ & 0.9878 & 1.286 & -1.529 & -2.447 & 1.3 & \multirow{5}{*}{$0.1-2.0$} \\
\hline & $h_{2}$ & 1.0043 & 1.4303 & -103.975 & -2.692 & 1.4 & \\
\hline & $h_{3}$ & 1.1037 & 0.7056 & -3.097 & -5.611 & 1.6 & \\
\hline & $h_{4}$ & 1.0228 & 1.8667 & 65.727 & -2.988 & 2.0 & \\
\hline & $p$ & & & & & 2.9 & \\
\hline \multirow{5}{*}{$\mathrm{Mg}\left(\mathrm{ClO}_{4}\right)_{2}$} & $h_{1}$ & 1.0142 & 1.3461 & -1.508 & -2.85 & 1.0 & \multirow{5}{*}{$0.1-0.2$} \\
\hline & $h_{2}$ & 1.0278 & 1.4968 & -124.41 & -3.080 & 1.2 & \\
\hline & $h_{3}$ & 1.1196 & 0.7476 & -2.957 & -5.961 & 1.7 & \\
\hline & $h_{4}$ & 1.043 & 1.933 & 75.732 & -3.352 & 2.0 & \\
\hline & $p$ & & & & & 1.9 & \\
\hline \multirow{5}{*}{$\mathrm{Mg}\left(\mathrm{NO}_{3}\right)_{2}$} & $h_{1}$ & 0.9849 & 1.1643 & -0.927 & -2.323 & 1.1 & \multirow{5}{*}{$0.1-2.0$} \\
\hline & $h_{2}$ & 0.9966 & 1.3079 & -42.667 & -2.489 & 1.4 & \\
\hline & $h_{3}$ & 1.0582 & 0.6573 & -1.632 & -4.042 & 1.6 & \\
\hline & $h_{4}$ & 1.0095 & 1.7555 & 28.735 & -2.683 & 1.8 & \\
\hline & $p$ & & & & & 2.2 & \\
\hline \multirow{5}{*}{$\mathrm{MgAc}_{2}$} & $h_{1}$ & 0.946 & 1.01 & -0.5577 & -1.766 & 2.5 & \multirow{5}{*}{$0.1-4.0$} \\
\hline & $h_{2}$ & 0.9667 & 1.225 & -18.031 & -1.994 & 1.4 & \\
\hline & $h_{3}$ & 1.005 & 0.6129 & -0.8487 & -2.7667 & 1.9 & \\
\hline & $h_{4}$ & 0.9853 & 1.783 & 14.678 & -2.214 & 0.9 & \\
\hline & $p$ & & & & & - & \\
\hline
\end{tabular}


(Table 2). Continued.

\begin{tabular}{|c|c|c|c|c|c|c|c|}
\hline Electrolyte & Model & $k_{1}$ & $k_{2}$ & $\alpha_{1}$ & $\alpha_{2}$ & $\sigma / 10^{3}$ & $\begin{array}{l}\text { Molality } \\
/ \mathrm{mol}^{-1} \mathbf{k g}^{-1}\end{array}$ \\
\hline \multirow{5}{*}{$\mathrm{NiCl}_{2}$} & $h_{1}$ & 0.9808 & 1.2833 & --0.941 & -2.291 & 1.1 & \multirow{5}{*}{$0.1-5.0$} \\
\hline & $h_{2}$ & 0.9966 & 1.461 & -67.608 & -2.504 & 1.0 & \\
\hline & $h_{3}$ & 1.067 & 0.7456 & -1.653 & -4.172 & 1.2 & \\
\hline & $h_{4}$ & 1.0119 & 1.9326 & 44.189 & -2.725 & 1.8 & \\
\hline & $p$ & & & & & & \\
\hline \multirow{5}{*}{$\mathrm{Na}_{2} \mathrm{CO}_{3}[25]$} & $h_{1}$ & 0.9455 & 2.6776 & -0.0268 & -1.8203 & 0.8 & \multirow{5}{*}{$0.1-2.7$} \\
\hline & $h_{2}$ & 0.9463 & 3.04 & -199.99 & -1.827 & 0.6 & \\
\hline & $h_{3}$ & 0.9517 & 1.799 & -0.033 & -1.879 & 0.3 & \\
\hline & $h_{4}$ & 0.9464 & 3.497 & 71.0 & -1.828 & 0.6 & \\
\hline & $p$ & & & & & 5.7 & \\
\hline \multirow{5}{*}{$\mathrm{Na}_{2} \mathrm{SO}_{4}$} & $h_{1}$ & 0.9184 & 2.495 & -0.0246 & -1.572 & 2.1 & \multirow{5}{*}{$0.1-4.0$} \\
\hline & $h_{2}$ & 0.9212 & 2.9897 & -135.1 & -1.591 & 1.6 & \\
\hline & $h_{3}$ & 0.9262 & 1.795 & -0.025 & -1.633 & 1.2 & \\
\hline & $h_{4}$ & 0.9229 & 3.543 & 57.0 & -1.603 & 1.4 & \\
\hline & $p$ & & & & & 2.4 & \\
\hline \multirow{5}{*}{$\left(\mathrm{NH}_{4}\right)_{2} \mathrm{SO}_{4}$} & $h_{1}$ & 0.9206 & 1.533 & -0.066 & -1.535 & 2.4 & \multirow{5}{*}{$0.1-5.5$} \\
\hline & $h_{2}$ & 0.9265 & 1.94 & -15.166 & -1.581 & 1.5 & \\
\hline & $h_{3}$ & 0.9356 & 1.065 & -0.0728 & -1.673 & 1.7 & \\
\hline & $h_{4}$ & 0.9295 & 2.543 & 9.634 & -1.605 & 0.9 & \\
\hline & $p$ & & & & & 3.2 & \\
\hline \multirow{5}{*}{$\mathrm{K}_{2} \mathrm{HPO}_{4}[29]$} & $h_{1}$ & 0.9434 & 2.4229 & -0.02943 & -1.775 & 0.1 & \multirow{5}{*}{$0.17-2.0$} \\
\hline & $h_{2}$ & 0.9437 & 2.6532 & -75.238 & -1.7776 & 0.1 & \\
\hline & $h_{3}$ & 0.9503 & 1.5034 & -0.0413 & -1.8413 & 0.1 & \\
\hline & $h_{4}$ & 0.944 & 3.081 & 28.1552 & -1.7802 & 0.04 & \\
\hline & $p$ & & & & & $4.1^{\mathrm{a}}$ & \\
\hline \multirow{5}{*}{$\mathrm{Pb}\left(\mathrm{NO}_{3}\right)_{2}$} & $h_{1}$ & 0.9779 & 0.062 & 3.215 & -4.8 & 3.2 & \multirow{5}{*}{$0.1-2.0$} \\
\hline & $h_{2}$ & 1.011 & 0.017 & 17.986 & -18.848 & 3.0 & \\
\hline & $h_{3}$ & 0.9567 & 0.0625 & 2.515 & -1.488 & 3.2 & \\
\hline & $h_{4}$ & 1.0238 & 0.5592 & -4.672 & -3.279 & 1.6 & \\
\hline & $p$ & & & & & 1.6 & \\
\hline \multirow{5}{*}{$\mathrm{UO}_{2}\left(\mathrm{ClO}_{4}\right)_{2}$} & $h_{1}$ & 1.0308 & 1.3521 & -2.045 & -3.166 & 2.5 & \multirow{5}{*}{$0.1-2.5$} \\
\hline & $h_{2}$ & 1.0483 & 1.5025 & -172.96 & -3.496 & 3.5 & \\
\hline & $h_{3}$ & 1.153 & 0.724 & -4.356 & -7.505 & 2.2 & \\
\hline & $h_{4}$ & 1.0666 & 1.935 & 105.04 & -3.869 & 4.7 & \\
\hline & $p$ & & & & & 2.2 & \\
\hline
\end{tabular}

${ }^{\mathrm{a}}$ This datum is according to Extended Pitzer equation.

various effects, such as the Coulomb's force between ions, the action of ions with solvent particles, the higher-order interactions, even the deformation and polarization of ions, hydrogen bond, etc., giving a comprehensive upshot that could govern the thermodynamic behavior of electrolytes. It could be considered to be a dominant factor that represents the whole effects influencing electrolyte behavior.

Certainly, the term, $I^{k_{2}}$ could be also seen to be a distribution of all ions in the ion atmosphere. In DebyeHückel theory, a binomial is taken into account as the 
Table 3: Parameters and the Errors for Prediction of the Osmotic Coefficients of Triunivalent Electrolytes by the $h$ Function Model

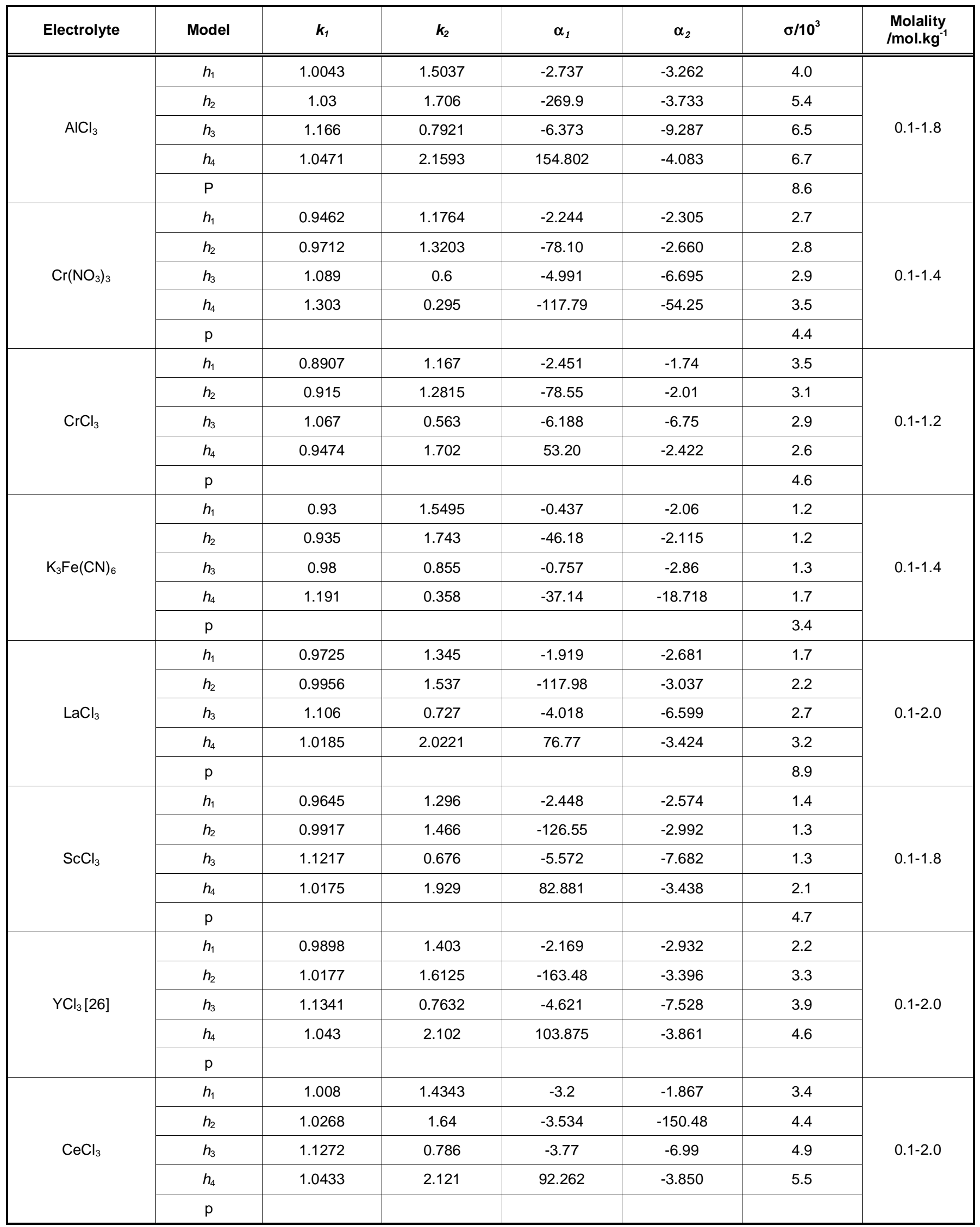


(Table 3). Continued.

\begin{tabular}{|c|c|c|c|c|c|c|c|}
\hline Electrolyte & Model & $k_{1}$ & $k_{2}$ & $\alpha_{1}$ & $\alpha_{2}$ & $\sigma / 10^{3}$ & $\begin{array}{l}\text { Molality } \\
/ \mathrm{mol}^{-1 . k g}\end{array}$ \\
\hline \multirow{5}{*}{$\mathrm{PrCl}_{3}$} & $h_{1}$ & 0.9662 & 1.3509 & -1.927 & -2.591 & 1.9 & \multirow{5}{*}{$0.1-2.0$} \\
\hline & $h_{2}$ & 0.9921 & 1.5495 & -122.44 & -2.9785 & 1.5 & \\
\hline & $h_{3}$ & 1.104 & 0.729 & -4.082 & -6.555 & 1.6 & \\
\hline & $h_{4}$ & 1.012 & 2.023 & 77.76 & -3.309 & 2.0 & \\
\hline & $p$ & & & & & & \\
\hline \multirow{5}{*}{$\mathrm{EuCl}_{3}$} & $h_{1}$ & 0.9822 & 1.389 & -2.0287 & -2.841 & 2.6 & \multirow{5}{*}{$0.1-2.0$} \\
\hline & $h_{2}$ & 1.0051 & 1.5861 & -142.62 & -3.211 & 3.7 & \\
\hline & $h_{3}$ & 1.1186 & 0.7504 & -4.299 & -7.039 & 4.1 & \\
\hline & $h_{4}$ & 1.0266 & 2.0678 & 90.302 & -3.591 & 5.0 & \\
\hline & $p$ & & & & & & \\
\hline
\end{tabular}

Table 4: Parameters and the Errors for Prediction of the Osmotic Coefficients of Tetraunivalent Electrolytes by the $h$ Function Model

\begin{tabular}{|c|c|c|c|c|c|c|c|}
\hline Electrolyte & Model & $k_{1}$ & $k_{2}$ & $\alpha_{1}$ & $\alpha_{2}$ & $\sigma / 10^{3}$ & $\begin{array}{l}\text { Molality } \\
/ \mathrm{mol}^{-} \mathrm{kg}^{-1}\end{array}$ \\
\hline \multirow{5}{*}{$\mathrm{K}_{4} \mathrm{Fe}(\mathrm{CN})_{6}$} & $h_{1}$ & 0.9163 & 4.6 & -0.256 & -2.022 & 0.4 & \multirow{5}{*}{$0.1-0.9$} \\
\hline & $h_{2}$ & 0.9163 & 4.921 & -54300 & -2.022 & 0.4 & \\
\hline & $h_{3}$ & 0.92 & 10.67 & 0.393 & -2.054 & 1.1 & \\
\hline & $h_{4}$ & 1.2243 & 0.4102 & -43.531 & -20.801 & 1.9 & \\
\hline & $p$ & & & & & 9.9 & \\
\hline \multirow{5}{*}{$\mathrm{Th}\left(\mathrm{NO}_{3}\right)_{4}$} & $h_{1}$ & 0.7826 & 1.0221 & -2.031 & -1.037 & 6.1 & \multirow{5}{*}{$0.1-2.0$} \\
\hline & $h_{2}$ & 0.8754 & 1.1996 & -41.969 & -1.757 & 3.9 & \\
\hline & $h_{3}$ & 1.0012 & 0.5029 & -5.065 & -5.29 & 4.8 & \\
\hline & $h_{4}$ & 0.9499 & 1.7495 & 36.532 & -2.616 & 1.5 & \\
\hline & $p$ & & & & & & \\
\hline
\end{tabular}

Table 5: Parameters and the Errors for Prediction of the Osmotic Coefficients of Bibivalent Electrolytes by the $h$ Function Model

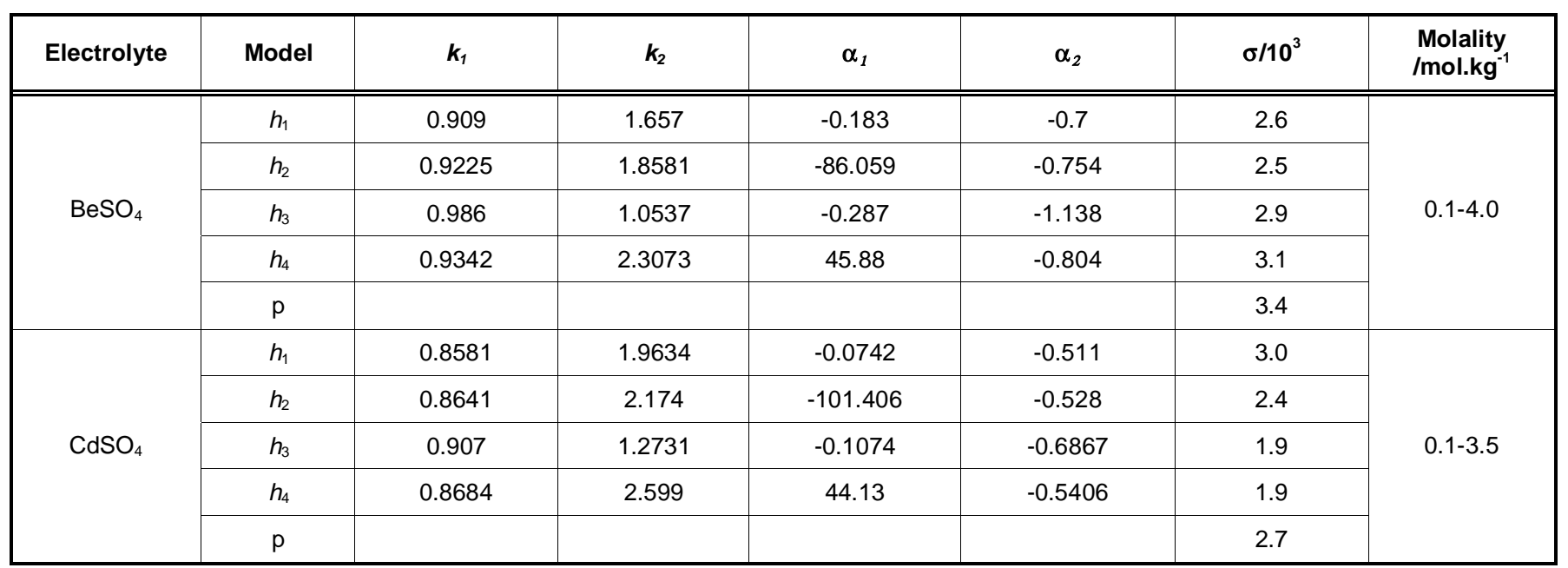


(Table 5). Continued.

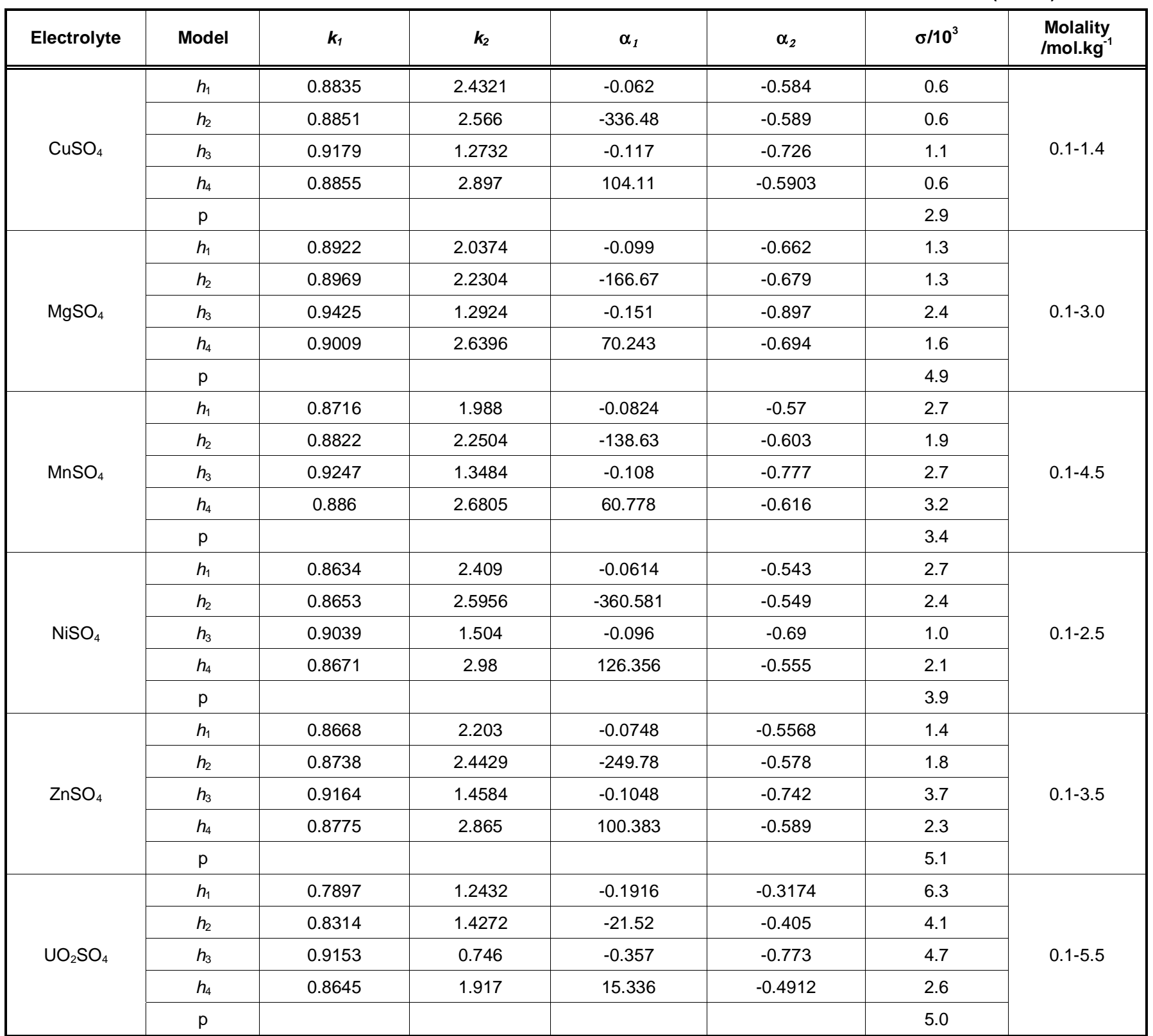

Table 6: Parameters and the Errors for Prediction of the Osmotic Coefficients of Tribivalent Electrolytes by the $h$ Function Model

\begin{tabular}{|c|c|c|c|c|c|c|c|}
\hline Electrolyte & Model & $k_{1}$ & $k_{2}$ & $\alpha_{1}$ & $\alpha_{2}$ & $\sigma / 10^{3}$ & $\begin{array}{l}\text { Molality } \\
\text { /mol.kg }^{-1}\end{array}$ \\
\hline \multirow{3}{*}{$\mathrm{Al}_{2}\left(\mathrm{SO}_{4}\right)_{3}$} & $h_{1}$ & 0.7426 & 2.0243 & -2.033 & -0.612 & 3.6 & \multirow{3}{*}{$0.1-1.0$} \\
\hline & $h_{3}$ & 1.1386 & 0.7929 & -10.21 & -7.086 & 2.4 & \\
\hline & $h_{4}$ & 0.7827 & 2.5826 & 211.388 & -0.749 & 3.0 & \\
\hline \multirow{3}{*}{$\mathrm{Cr}_{2}\left(\mathrm{SO}_{4}\right)_{3}$} & $h_{2}$ & 0.9084 & 2.2632 & -637.094 & -1.342 & 1.4 & \multirow{3}{*}{$0.1-1.2$} \\
\hline & $h_{3}$ & 1.1905 & 0.9026 & -8.336 & -7.281 & 1.8 & \\
\hline & $h_{4}$ & 0.9223 & 2.6843 & 276.32 & -1.436 & 1.1 & \\
\hline
\end{tabular}


Table 7: Parameters and Errors for Reproduction of the Relative Molal Vapor Pressure Lowering, $\delta$ and the Equivalent Conductivity, $\rho$ of $\mathrm{KCl}$ Solution at $298 \mathrm{~K}$ by the $h$ Function Model

\begin{tabular}{|c|c|c|c|c|c|c|c|c|}
\hline Properties & Model & $k_{1}$ & $k_{2}$ & $\alpha_{1}$ & $\alpha_{2}$ & $\sigma$ & $A R D^{c}$ & $\begin{array}{l}\text { Molality } \\
/ \mathrm{mol}^{-k^{-1}}\end{array}$ \\
\hline \multirow{3}{*}{$\delta$} & $h_{1}$ & 0.9732 & 1.0674 & $-2.938 \times 10^{-3}$ & -0.05731 & $1.8 \times 10^{-5}$ & 0.0004 & \multirow{3}{*}{$0.1-4.8$} \\
\hline & $h_{3}$ & 0.98554 & 0.71065 & $-3.508 \times 10^{-3}$ & -0.06278 & $1.3 \times 10^{-5}$ & 0.0003 & \\
\hline & $h_{4}$ & 0.9807 & 1.7972 & 0.1273 & -0.05991 & $1.6 \times 10^{-5}$ & 0.0004 & \\
\hline \multirow{2}{*}{$\rho$} & $h_{3}$ & 1.0066 & 0.089 & 278.081 & -222.073 & 0.6 & 0.0003 & \multirow{2}{*}{$0.0005-0.1$} \\
\hline & $h_{4}$ & 1.0074 & 0.4229 & -290.17 & -333.681 & 0.3 & 0.0002 & \\
\hline
\end{tabular}

${ }^{\mathrm{C}} A R D=\frac{\sum_{1}^{N}|Q-Q \mathrm{cal}| / Q}{N}$ where $N$ is the number of data points, $Q$ and $Q_{\mathrm{cal}}$ are, respectively, $\delta$ or $\rho$ determined experimentally and calculated by the $h$ function model.

Table 8: Parameters and the Errors for Reproduction of the Solubility of $\mathrm{MgCl}_{2}$ in $\mathrm{HCl}-\mathrm{LiCl}-\mathrm{MgCl}_{2}-\mathrm{H}_{2} \mathrm{O}$ System at 293K by the $h$ Function Model

\begin{tabular}{|c|c|c|c|c|c|c|}
\hline Model & $\boldsymbol{k}_{\mathbf{1}}$ & $\boldsymbol{k}_{\mathbf{2}}$ & $\boldsymbol{\alpha}_{1}$ & $\boldsymbol{\alpha}_{2}$ & $\boldsymbol{\sigma}$ & ARD \\
\hline \hline$h_{1}$ & 1.0 & 1.121 & 1.313 & -91.216 & 0.064 & 0.016 \\
\hline$h_{2}$ & 0.978 & 1.7165 & 303.721 & -82.967 & 0.065 & 0.016 \\
\hline$h_{3}$ & 0.9977 & 0.834 & 1.168 & -88.841 & 0.064 & 0.016 \\
\hline$h_{4}$ & 1.041 & 1.8 & -215.101 & -104.122 & 0.075 & 0.019 \\
\hline BET [32] & & & & & 0.091 & 0.024 \\
\hline
\end{tabular}

radial distribution function of ionic atmosphere surrounding a centre ion. Pitzer extended it to a trinomial, constructing a quadratic equation of the molal concentration of solute. According to the statistic physics, the properties of system are dependent on the distribution of particles. The characteristics of electrolytes could be indentified with the aid of $I^{k_{2}}$. In this paper, the term, $m^{k_{2}}$ is used instead of $I^{k_{2}}$, this is because the ionic strength is directly proportional to $m^{k_{2}}$ for each kind of the single electrolyte. Therefore, $m^{k_{2}}$ is used to replace $I^{k_{2}}$ for all single electrolytes.

In the $\omega$ function, $k_{1}$ is a constant to be confirmed. In most cases, $k_{1}$ is close to one. As the valence of electrolyte or the ion strength increases, $k_{1}$ deviates from one further. For the high-dilute uniunivalent electrolytes, $k_{1}$ is more close to one. The deviation of $k_{1}$ from one could be used to judge the ideality of electrolyte solution. Due to the total effects of all forces concerning a lot of factors, the regularity of the change in $k_{2}$ is not followed.

It should be pointed out that the linear rule of $\omega$ against $m^{k_{2}}$ exists only in a given concentration interval. For a certain electrolyte from the high-dilute to the saturated solution, the entire concentration range is too wide to expect one linear equation of $\omega$ against $m^{k_{2}}$ to resolve all problems. Here, it needs to section whole range into two or three concentration subintervals. In every subinterval, there is a corresponding linear relation of $\omega$ against $m^{k_{2}}$. This just means that the increase in concentration would alter the total interionic interactions, and makes the electrolyte deviate ideality more far. As the concentration increases, $k_{1}$ would be gradually far from one. Take $\mathrm{HNO}_{3}$ for example. The concentration range is from 0.001 to 28 molal, and the osmotic coefficients are taken form Ref. [21]. The entire concentration range is divided into three subintervals, i.e. 0.001 to $0.1,0.1$ to 12 and 12 to 28 in molality. And construct three linear equations of $\omega$ against $m^{k_{2}}$ in these subintervals, respectively. The parameters are listed in Table 9, and the reproduced results are in Figure 2. Obviously, the narrower the subintervals are taken, the more accurate the reproduced results will be.

Many properties of electrolyte solution, as shown above, can be reproduced by Eq. (6) as long as $\Phi$ is 
Table 9: Parameters and the Errors for Prediction of the Osmotic Coefficients of $\mathrm{HNO}_{3}$ by the $h_{1}$. Function Model

\begin{tabular}{|c|c|c|c|c|c|c|}
\hline Subinterval $/ \boldsymbol{m}$ & $\boldsymbol{k}_{\mathbf{1}}$ & $\boldsymbol{k}_{\mathbf{2}}$ & $\boldsymbol{\alpha}_{1}$ & $\boldsymbol{\alpha}_{2}$ & $\boldsymbol{\sigma}$ & ARD \\
\hline \hline $0.001-0.1$ & 1.021 & 0.01 & 7.5173 & -9.469 & 0.001 & 0.0008 \\
\hline $0.1-12.0$ & 0.9633 & 0.8763 & -0.294 & -1.503 & 0.003 & 0.002 \\
\hline $12.0-28.0$ & 0.46 & 0.01 & -206.879 & 209.863 & 0.004 & 0.002 \\
\hline
\end{tabular}

replaced by corresponding properties, such as $\delta, \rho$, etc., showing the these properties are also linearly related to the ionic strength or the molal concentration exponential for single electrolytes.

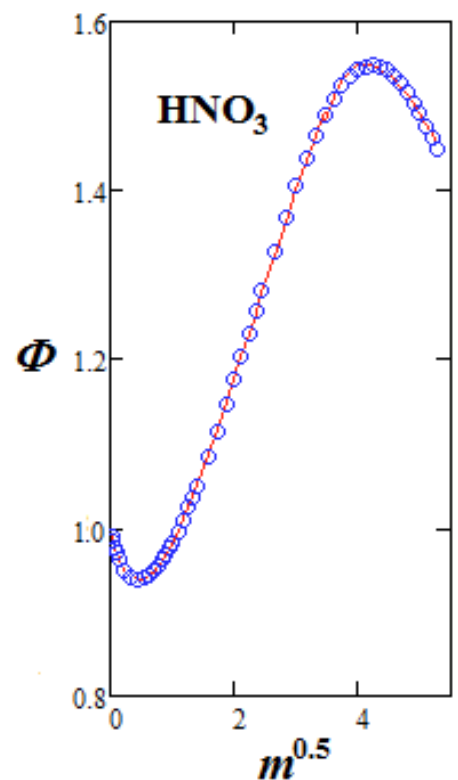

Figure 2: Prediction of the osmotic coefficients for $\mathrm{HNO}_{3}$ by section of whole concentration interval into three subintervals (0.001-0.1, 0.1-12 and 12-28 molality).

$\circ$, experimental point; —_, model.

Because of the equivalence of $m$ and $x$, construct $h_{2}=x^{k_{2}}$. We discover that the potential $\omega$ is also linearly related to $h_{2}$ for a various valent type of electrolytes. If taking 'the Shannon information entropy' of $h_{1}$ or $h_{2}$ [33] i.e. $h_{3}=h_{1} \ln \left(h_{1}\right)$ or $h_{4}=h_{2} \ln \left(h_{2}\right)$. The function $\omega$ is also linearly related to $h_{3}$ and $h_{4}$, respectively. The calculation parameters and errors are listed in Tables 1-8, respectively. According to the errors, we can see that the results by the present model are better than those by Pitzer for most of uniunivalent, biunivalent, triunivalent, tetraunivalent and bibivalent electrolytes, indicating that the linear rule is feasible to predict some properties for electrolytic solutions.

It should be pointed out that, at first glance, it seems that there are four parameters $k_{1}, k_{2}, \alpha_{1}$ and $\alpha_{2}$ to describe the properties of system, but $\alpha_{1}$ and $\alpha_{2}$ are not dependent. They are automatically determined as $k_{1}$ and $k_{2}$ are optimized and attained.

\section{SUMMARY}

As is well-known, all thermodynamic properties of an electrolyte solution must be a comprehensive effect of the interactions of various particles in the solution. It relates to all kinds of potentials of these particles, such as those between ions, between solvent and ions, between solvent particles, and their interactions. This could be represented by a dimensionless potential $\omega$. It, substantially, is an osmotic coefficient on mole fraction base. The potential can integrate the effects of various particles, and describe the macroscopic properties of the electrolyte solution well.

The $h$ functions such as $m^{k_{2}}, x^{k_{2}}$ and their respective 'Shannon information entropies' $m^{k_{2}} \ln \left(m^{k_{2}}\right)$, and $x^{k_{2}} \ln \left(x^{k_{2}}\right)$ are all related to the micro-distribution of solute and solvent molecules. This never was taken into account before.

It is found that the dimensionless potential $\omega$ for expressing the integral interactions is linearly related to these $h$ functions, respectively. Based on this pertinence, the models are established for analytical expression of some thermodynamic and transferring properties of electrolytes, such as osmotic coefficient, activity of solvent, and relative molal vapor pressure lowering, equivalent conductivity and solubility of a salt in electrolytic mixture. The models are suitable for the electrolyte solutions with different valence.

\section{ACKNOWLEDGEMENT}

This research was financially supported by the National Natural Science Fund of China (No. 50874119).

\section{REFERENCES}

[1] Fang Z. Theory and application of thermoelectrochemistry in $<$ Thermodynamics-Physical Chemistry of Aqueous System> edited by Juan Carlos Moreno-Pirajan. Tech Press 2011; pp. 27-48. ISBN: 978-953-307-979-0

[2] Fang Z, Wang SF, Zhang ZH. The electrochemical Peltier heat of the standard hydrogen electrode reaction. Thermochimica Acta 2008; 473: 40-4. http://dx.doi.org/10.1016/.ttca.2008.04.002 
[3] Debye P, Hückel E. The theory of electrolytes. I. Lowering of freezing point and related phenomena. Physik Zeitschrift 1923; 24: 185-206.

[4] Castellan GW. Physical Chemistry 3rd Ed. Benjamin Cummings, Pub. Co 1983.

[5] Guggenheim EA. The specific thermodynamic properties of aqueous solutions of strong electrolytes. Phil Mag 1935; 19: 588-643.

http://dx.doi.org/10.1080/14786443508561403

[6] Stokes $\mathrm{RH}$, Robinson RA. Ionic hydration and activity in electrolyte solutions. J Am Chem Soc 1948; 70: 1870-8. http://dx.doi.org/10.1021/ja01185a065

[7] Pitzer KS. Thermodynamics of electrolytes. I. Theoretical basis and general equations. J Phys Chem 1973; 77: 268-77. http://dx.doi.org/10.1021/j100621a026

[8] Pitzer KS, Kim JJ. Thermodynamics of electrolytes. IV. Activity and osmotic coefficients for mixed electrolytes. J Am Chem Soc 1974; 96: 5701-7.

http://dx.doi.org/10.1021/ja00825a004

[9] $\mathrm{Hu}$ YF. The thermodynamics of nonelectrolyte systems at constant activities of any number of components. J Phys Chem B 2003; 107: 13168-77. http://dx.doi.org/10.1021/jp035528f

[10] Patwardhan VS, Kumar A. Thermodynamic properties of aqueous solutions of mixed electrolytes: a new mixing rule. AIChE J 1993; 39: 711-4. http://dx.doi.org/10.1002/aic.690390422

[11] Young TF, Wu YC, Krawetz AA. Thermal effects of the interactions between ions of like charge. Discuss Faraday Soc $1957 ; 24: 37-42$.

http://dx.doi.org/10.1039/df9572400037

[12] Waisman E, Lebowitz JL. Exact solution of an integral equation for the structure of a primitive model of electrolytes. J Chem Phys 1970; 52: 4307-8.

http://dx.doi.org/10.1063/1.1673642

[13] Varela LM, Pérez-Rodríguez M, García M, Mosquera V. Thermodynamics of electrolyte solutions in the modified mean spherical approximation. J Chem Phys 2000; 113: 292-6. http://dx.doi.org/10.1063/1.481794

[14] Rasaiah JC, Card DN, Valleau JP. Calculations on the "restricted primitive model" for 1-1 electrolyte solutions. J Chem Phys 1972; 56: 248-55.

http://dx.doi.org/10.1063/1.1676854

[15] Harned HS, Owen BB. The Physical Chemistry of Electrolytic Solutions, 2nd Ed Reinhold Publ. Corp, New York, NY 1950.

[16] Robinson RA, Stokes RH. Electrolytic Solutions, 2nd Ed. Butterworths, Sci. Pub. London 1959.

[17] Bassett RL, Melchior DC. Chemical Modeling of Aqueous Systems, ACS, Washington, DC, 1990; pp. 1-14. http://dx.doi.org/10.1021/bk-1990-0416.ch001

[18] Varela LM, Garcia M, Mosquera V. Activity coefficients of electrolyte solutions in the modified mean spherical approximation. Physica A 2003; 323: 75-87.

http://dx.doi.org/10.1016/S0378-4371(03)00054-2

[19] Clegg SL, Pitzer KS, Brimblecombe P. Thermodynamics of multicomponent, miscible, ionic solutions. Mixtures including unsymmetrical electrolytes. J Phys Chem 1992; 96: 9470-9. http://dx.doi.org/10.1021/j100202a074

[20] Fang Z. A model describing the osmotic coefficients and the activities of water for electrolyte solutions. Asian $\mathrm{J}$ Chem 2011; 23: 5384-92.
[21] Hamer WJ, Wu YC. Osmotic coefficients and mean activity coefficients of uni-univalent electrolytes in water at $25{ }^{\circ} \mathrm{C} . \mathrm{J}$ Phys Chem Ref Data 1972; 1: 1047-99. http://dx.doi.org/10.1063/1.3253108

[22] Pitzer KS, Roy RN, Silvester LF. Thermodynamics of electrolytes. 7. Sulfuric acid. J Am Chem Soc 1977; 99: 4930-6.

http://dx.doi.org/10.1021/ja00457a008

[23] Rard JA. Isopiestic determination of the osmotic and activity coefficients of aqueous manganese (II) chloride, manganese(II) sulfate, and rubidium chloride at $25{ }^{\circ} \mathrm{C}$. J Chem Eng Data 1984; 29 : 443-50. http://dx.doi.org/10.1021/je00038a024

[24] Rard JA, Miller DG. Isopiestic determination of the osmotic and activity coefficients of aqueous cesium chloride, strontium chloride, and mixtures of sodium chloride and cesium chloride at 25 oC. J Chem Eng Data 1982; 27: 16973.

http://dx.doi.org/10.1021/je00028a021

[25] Rard JA, Miller DG. Isopiestic determination of the osmotic and activity coefficients of aqueous mixtures of sodium chloride and strontium chloride at $25{ }^{\circ} \mathrm{C}$. J Chem Eng Data 1982; 27: 342-6.

http://dx.doi.org/10.1021/je00029a033

[26] Peiper JC, Pitzer KS. Thermodynamics of aqueous carbonate solutions including mixtures of sodium carbonate bicarbonate, and chloride. J Chem Thermodyn 1982; 14: 613-8. http://dx.doi.org/10.1016/0021-9614(82)90078-7

[27] Rard JA, Spedding FH. Isopiestic determination of the activity coefficients of some aqueous rare-earth electrolyte solutions at $25{ }^{\circ} \mathrm{C}$. 6. Europium trinitrate, yttrium nitrate, yttrium chloride. J Chem Eng Data 1982; 27: 454-61. http://dx.doi.org/10.1021/je00030a026

[28] Robinson RA, Bower VE. Thermodynamics of the ternary system: Water-sodium chloride-barium chloride at $25^{\circ} \mathrm{C}[\mathrm{J}] . \mathrm{J}$ Res Natl Bur Stand 1965; 69A: 19. http://dx.doi.org/10.6028/jres.069A.004

[29] Robinson RA, Bower VE. An additivity rule for the vapor pressure lowering of aqueous solutions [J]. J Res Natl Bur Stand 1965; 69A: 365-7. http://dx.doi.org/10.6028/jres.069A.037

[30] Popovic DZ, Miladinovic J, Miladinovic ZP, Ivoševic BB, Todorovic MD, Rard JA. Isopiestic determination of the osmotic and activity coefficients of the $\left\{\mathrm{yKNO}_{3}+(1-\right.$ y) $\mathrm{K}_{2} \mathrm{HPO}_{4}$ (aq) system at $\mathrm{T}=298.15 \mathrm{~K}$. J Chem Thermodynam 2012; 55: 172-83.

[31] Hu K-Y, Cai W-Q. Experimental study on the solubility of the quaternary system $\mathrm{H}^{+}, \mathrm{Li}^{+}, \mathrm{Mg}^{2+} / \mathrm{Cl}^{-}-\mathrm{H}_{2} \mathrm{O}$ at $0{ }^{\circ} \mathrm{C}$. Acta Chim Sin 1965; 31: 189-97.

[32] Zeng $D$, Liu $H$, Chen $Q$. Simulation and prediction of solubility phase diagram for the separation of $\mathrm{MgCl}_{2}$ from $\mathrm{LiCl}$ brine using $\mathrm{HCl}$ as a salting-out agent. Hydrometallurgy 2007; 89: 21-31.

http://dx.doi.org/10.1016/j.hydromet.2007.05.001

[33] Shannon CE. A mathematical theory of communication. Bell Syst Tech J 1948; 27: 379-423.

http://dx.doi.org/10.1002/j.1538-7305.1948.tb01338.x

Received on 05-10-2015

Accepted on 20-11-2015

Published on 18-12-2015

\section{DOI: http://dx.doi.org/10.6000/1929-5030.2015.04.04.5}

(c) 2015 Zheng Fang; Licensee Lifescience Global.

This is an open access article licensed under the terms of the Creative Commons Attribution Non-Commercial License (http://creativecommons.org/licenses/by-nc/3.0/) which permits unrestricted, non-commercial use, distribution and reproduction in any medium, provided the work is properly cited. 